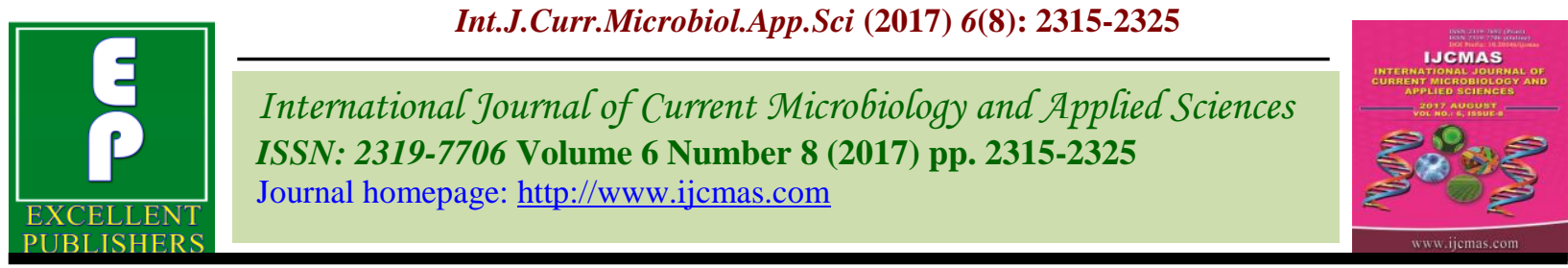

Original Research Article

https://doi.org/10.20546/ijcmas.2017.608.273

\title{
Effect of Tillage Practices and Supplemental Irrigation Options on Growth, Yield and Soil Biological Properties of Hybrid Maize
}

\author{
M. Saravanan* \\ Subject Matter Specialist (Agronomy), ICAR-Krishi Vigyan Kendra, Ariyalur District, \\ Tamil Nadu, India \\ *Corresponding author
}

A B S T R A C T

\begin{tabular}{|l|}
\hline Ke y w or d s \\
Crop residue, \\
Maize, \\
Supplemental \\
irrigation, Tillage \\
and microbes. \\
\hline Article Info \\
\hline $\begin{array}{l}\text { Accepted: } \\
\text { 21 June 2017 } \\
\text { Available Online: } \\
\text { 10 August } 2017\end{array}$ \\
\hline
\end{tabular}

Field experiment was conducted in two consecutive growing seasons at Agricultural Engineering College and Research Institute, Kumulur, Tamil Nadu to evaluate the tillage practices, crop residue and supplemental irrigation through drip irrigation in maize under rainfed condition. The experiment was laid out in strip plot design with three replications. The main plot treatments were conventional tillage, minimum tillage without crop residue, minimum tillage with crop residue @ $5 \mathrm{t} \mathrm{ha}^{-1}$ and minimum tillage with crop residue @ 10 $\mathrm{t} \mathrm{ha}^{-1}$. The sub plot treatments were control (without irrigation), supplemental drip irrigation 4, 6, 8 and 10 times during the cropping period. Tillage practices and crop residue with supplemental drip irrigation practices could potentially lead to significant difference in growth parameters, microbes populations, yield attributes and maximum yield $8345,8856 \mathrm{~kg} \mathrm{ha}^{-1}$ of maize in both the seasons by application of minimum tillage with crop residue $10 \mathrm{t} \mathrm{ha}^{-1}+$ supplemental drip irrigation ten times. Therefore, minimum tillage (one pass of mouldboard plough followed by two passes of disk harrow) with crop residue $10 \mathrm{t} \mathrm{ha}^{-1}+$ supplemental irrigation through drip irrigation ten times was found to be more appropriate and profitable to improving yield of maize under rainfed condition.

\section{Introduction}

Soil quality (SQ) highly depends on its structure, natural productivity, and human influence. Soil organisms are important elements for preserved ecosystem biodiversity and services thus assess functional and structural biodiversity in arable soils is interest. Main threats to soil biodiversity occurred by mechanical impacts (soil compaction, soil tillage) and chemical stress (plant protection measures) in agricultural management.

Tillage is one of the major management practices affecting soil physical parameters. The influence of tillage systems on the total soil organic matter (OM) content is detectable only after several years of its application.

Microbial activity may respond to disturbances on a shorter period of time than those based on physical or chemical properties. As a consequence, microbiological properties such as soil enzyme activities have been suggested as potential indicators of SQ (Saviozzi et al., 2001) because of their rapid response to changes in soil management (Kandeler et al., 1999). In many cases, both bacteria and fungi were more abundant under no-tillage than conventional tillage (Helgason et al., 2009). 
Tillage systems affect the soil physical and chemical environment in which soil organisms live, thereby affecting soil organisms in different ways (Klavdivko 2001). Numerous studies in temperate regions have shown that decreasing tillage intensity results in higher organic $\mathrm{C}$ and $\mathrm{N}$ and improved soil quality (SQ) (Soon et al., 2001). Conservation tillage practices (reduced or no-tillage) result in increasing enzyme activities (Acosta-Martinez et al., 2003), microbial biomass (Franzluebbers et al., 1995), and fungal and bacteria dominance under NT (Helgason et al., 2009). Suitable soil management can be practiced through conservation tillage (including zero tillage), high crop residue return and crop rotation. Minimum-tillage (MT) is the most adapted conservation tillage system, which involves minimal disturbance of the surface residue.

Cropping systems that return crop residues to the field significantly increase the activity of a wide range of soil enzymes, compared to unamended soils, due to the stimulation of microbial activity (Frey et al., 1999).

In dry areas mostly shortage of soil moisture occurs during the most sensitive growth stages like flowering and grain filling stage of the crops. As a resulting is poor crop growth and yield. Supplemental irrigation (SI), with limited amount of water, when applied during the critical crop growth stages, can result in substantial improvement in yield and water productivity. Hence SI is an effective method to alleviate the adverse impact of soil moisture stress during dry spells on the yield of rainfed crops (Oweis and Hachum, 2006).

Maize (Zea mays L.) is the third most important cereal next to rice and wheat, in the world as well in India. It may be a substitute over the other dominant cropping system and may fulfill the future demand of human and animal feed. In order to increase production further there is no other option except to increase productivity by using available resources most efficiently.

Keeping this in view, the present investigation was undertaken to study the combined effect of tillage, crop residue incorporation and supplemental irrigation methods on the growth, biological population and yield of maize in Tamil Nadu.

\section{Materials and Methods}

A field experiment was carried out for two consecutive growing seasons at Agricultural Engineering College and Research Institute, Kumulur, Tamil Nadu. The experimental site is geographically situated at $10.56^{\circ}$ North latitude and $78.49^{\circ}$ East longitudes and at an altitude of $78 \mathrm{~m}$ above MSL. The soil was sandy loam in texture with $\mathrm{pH}$ 7.71. The fertility status of the soil was low, medium and high in the available $\mathrm{N}, \mathrm{P}_{2} \mathrm{O}_{5}$, and $\mathrm{K}_{2} \mathrm{O}$, with the values of 212,20 and $575 \mathrm{~kg} \mathrm{ha}^{-1}$ respectively. The main plot treatments were conventional tillage, minimum tillage without crop residue, minimum tillage with crop residue@ $5 \mathrm{t} \mathrm{ha}^{-1}$ and minimum tillage with crop residue@10 t ha ${ }^{-1}$. The sub plot treatments were (without irrigation) control, supplemental drip irrigation 4, 6, 8 and 10 times during the cropping period. The experiment was laid out in a strip plot design with three replications.

Conventional tillage included one pass of mouldboard plough to a depth of $15 \mathrm{~cm}$ and was followed by two passes of disk harrowing. Minimum tillage included only one pass of disk harrowing. The treatments were carried out on the same plots in the growing seasons. In both growing seasons, one of the most commercial maize NK6240 was sowing manually on paired row spacing of $60+30 \times 20 \mathrm{~cm}$ (totally there were two rows per plot). Before sowing a uniform fertilizer schedule was followed at the rate of 135:62.5:50 kg of $\mathrm{N}, \mathrm{P}_{2} \mathrm{O}_{5}$ and $\mathrm{K}_{2} \mathrm{O} \mathrm{ha}^{-1}$. 
Nitrogen was applied in three splits as 25: 50: 25 per cent as basal, at 25 and 50 DAS, respectively. The entire dose of phosphorus was applied basally. The potassium was applied in two equal split doses viz., basal and at 50 DAS.

Atrazine @ $0.5 \mathrm{~kg}$ a.i ha ${ }^{-1}$ was also applied for weed control after sowing of maize seed. During the growing season, the insecticides and fungicides were applied according to recommendations by the state agricultural university (SAU). All other necessary operations except those under study were kept normal and uniform for all the treatments.

The supplemental irrigation was given to the crop at the time of moisture stress period, which was determined based on the visual symptom (Wilting of plants). In study period four, six, eight and ten supplemental irrigations were given at various time period. The water was pumped by motor from farm pond and supplied to crops through drip irrigation system at a depth of $3 \mathrm{~cm}$.

Observations on growth characters such as plant height and dry matter production were recorded at 30, 60, 90 DAS and at harvest from five randomly selected plants in each plot.

The samples were collected from sampling rows in each plot for dry matter production and were used for the estimation of DMP. The population density of bacteria, fungi and actinomycetes were enumerated using serial dilution plate technique. The data on yield parameters and yield were also recorded.

\section{Results and Discussion}

\section{Effect of treatments on plant height}

Growth and development in plants are a consequence of excellent coordination of several processes operating at different growth stages of plant. The growth of maize influenced by various tillage treatments has been elucidated through the positive response on plant height.

The growth parameters of maize were significantly influenced by tillage and crop residue with supplemental irrigation through drip irrigation. The growth parameters were not influenced by treatments at 30 DAS. Among tillage and crop residue treatments, minimum tillage with crop residue $10 \mathrm{tha}^{-1}$ recorded significantly higher plant height (Table 1) $(164,198 \mathrm{~cm}$ and $159,191 \mathrm{~cm}$ at 60 DAS and at harvest during 2012 and 2013, respectively). Regarding irrigation practices, supplemental irrigation at 10 times was recorded higher plant height than without supplemental irrigation plot.

With regard to interaction effect, in a given tillage with crop residue treatment and supplemental drip irrigation, minimum tillage with crop residue $10 \mathrm{tha}^{-1}+$ supplemental drip irrigation 10 times registered distinctly higher plant height at 60 DAS and at harvest during both the years.

\section{Effect of treatments on dry matter production}

Tillage and crop residue with supplemental irrigation through drip irrigation significantly influenced the dry matter production (Tables 2 and 3). Among tillage and crop residue treatments, minimum tillage with crop residue $10 \mathrm{t} \mathrm{ha}^{-1}$ recorded significantly higher dry matter production $\left(8185,10634 \mathrm{~kg} \mathrm{ha}^{-1}\right.$ and $8166,10504 \mathrm{~kg} \mathrm{ha}^{-1}$ ) at 60 DAS and at harvest during 2012 and 2013, respectively. Among irrigation practices, supplemental irrigation at 10 times was recorded higher dry matter production in respective stages during both the seasons and it was comparable with supplemental irrigation at 8 times was recorded higher dry matter production than that in without supplemental irrigation plot. 
Table.1 Effect of tillage, crop residue and supplemental drip irrigation on plant height $(\mathrm{cm})$ of maize

\section{2}

\begin{tabular}{|c|c|c|c|c|c|c|c|c|c|c|c|c|c|c|c|c|c|c|c|c|}
\hline \multirow{2}{*}{$\begin{array}{c}\text { Treat } \\
\text { ment } \\
\text { s }\end{array}$} & \multicolumn{5}{|c|}{60 DAS } & \multicolumn{5}{|c|}{ At harvest } & \multicolumn{5}{|c|}{60 DAS } & \multicolumn{5}{|c|}{ At harvest } \\
\hline & $\mathbf{M}_{1}$ & $\mathbf{M}_{2}$ & $\mathbf{M}_{3}$ & $\mathbf{M}_{4}$ & Mean & $\mathbf{M}_{1}$ & $\mathbf{M}_{2}$ & $\mathbf{M}_{3}$ & $\mathbf{M}_{4}$ & Mean & $\mathbf{M}_{1}$ & $\mathbf{M}_{2}$ & $\mathbf{M}_{3}$ & $\mathbf{M}_{4}$ & Mean & $\mathbf{M}_{1}$ & $\mathbf{M}_{2}$ & $\mathbf{M}_{3}$ & $\mathbf{M}_{4}$ & Mean \\
\hline $\mathbf{S}_{1}$ & 133 & 136 & 139 & 143 & 138 & 160 & 165 & 169 & 175 & 167 & 128 & 134 & 139 & 144 & 137 & 154 & 159 & 162 & 168 & 161 \\
\hline $\mathbf{S}_{2}$ & 138 & 142 & 144 & 155 & 145 & 170 & 173 & 189 & 185 & 179 & 134 & 139 & 146 & 149 & 142 & 169 & 163 & 172 & 174 & 170 \\
\hline $\mathbf{S}_{\mathbf{3}}$ & 141 & 149 & 155 & 164 & 152 & 173 & 181 & 200 & 192 & 186 & 139 & 143 & 155 & 157 & 149 & 170 & 170 & 180 & 185 & 177 \\
\hline $\mathbf{S}_{5}$ & 152 & 166 & 171 & 182 & 168 & 187 & 195 & 213 & 235 & 208 & 156 & 157 & 170 & 180 & 166 & 188 & 194 & 203 & 226 & 203 \\
\hline Mean & 142 & 150 & 154 & 164 & & 174 & 180 & 194 & 198 & & 141 & 145 & 155 & 159 & & 171 & 174 & 182 & 191 & \\
\hline
\end{tabular}

\begin{tabular}{ccccccccc}
\hline & S Ed & CD $(\mathbf{P}=\mathbf{0 . 0 5})$ & S Ed & CD $(\mathbf{P}=\mathbf{0 . 0 5})$ & S Ed & CD (P=0.05) & S Ed & CD (P=0.05) \\
\hline M & 5.1 & 12.4 & 6.9 & 17.0 & 3.7 & 9.1 & 13.4 \\
S & 4.4 & 10.2 & 6.9 & 15.8 & 4.2 & 9.8 & 15.1 \\
M at S & 6.2 & 14.7 & 8.4 & 20.3 & 4.3 & 10.1 & 7.0 \\
S at M & 5.6 & 13.0 & 8.4 & 19.5 & 4.7 & 10.9 & 7.8 \\
\hline
\end{tabular}

$\mathrm{M}_{1}$ - Conventional tillage

$\mathrm{M}_{2}$ - Minimum tillage without crop residue

$\mathrm{M}_{3}$ - Minimum tillage with crop residue @ $5 \mathrm{~S}_{2}$ - Supplemental drip irrigation four times

tons ha- ${ }^{1}$
$\mathrm{M}_{4}$ - Minimum tillage with crop residue @ 10 tons ha- $^{1}$

$\mathrm{S}_{1}$-Control
$\mathrm{S}_{3}$ - Supplemental drip irrigation six times

$\mathrm{S}_{4}$ - Supplemental drip irrigation eight times

$\mathrm{S}_{5}$ - Supplemental drip irrigation ten times 
Table.2 Effect of tillage, crop residue and supplemental drip irrigation on dry matter production $\left(\mathrm{kg} \mathrm{ha}^{-1}\right)$ of maize 2012

\begin{tabular}{|c|c|c|c|c|c|c|c|c|c|c|}
\hline \multirow{2}{*}{ Treatments } & \multicolumn{5}{|c|}{60 DAS } & \multicolumn{5}{|c|}{ At harvest } \\
\hline & $\mathbf{M}_{1}$ & $\mathbf{M}_{2}$ & $\mathbf{M}_{3}$ & $\mathbf{M}_{4}$ & Mean & $M_{1}$ & $\mathbf{M}_{2}$ & $\mathbf{M}_{3}$ & $\mathbf{M}_{4}$ & Mean \\
\hline $\mathbf{S}_{1}$ & 5926 & 5896 & 6345 & 6645 & 6203 & 7963 & 7793 & 8025 & 8324 & 8026 \\
\hline $\mathbf{S}_{2}$ & 6745 & 6458 & 6942 & 7014 & 6789 & 8623 & 8124 & 9125 & 9624 & 8874 \\
\hline $\mathbf{S}_{\mathbf{3}}$ & 7156 & 6924 & 7468 & 7645 & 7298 & 9215 & 8745 & 10542 & 10457 & 9739 \\
\hline $\mathbf{S}_{4}$ & 7724 & 7745 & 8102 & 8867 & 8109 & 9956 & 9456 & 11108 & 11526 & 10511 \\
\hline $\mathbf{S}_{5}$ & 8541 & 8456 & 9023 & 10754 & 9193 & 10845 & 10245 & 12125 & 13242 & 11614 \\
\hline \multirow[t]{6}{*}{ Mean } & 7218 & 7096 & 7576 & 8185 & & 9320 & 8872 & 10185 & 10634 & \\
\hline & & S Ed & CD & 05) & & S Ed & & $\mathrm{CD}(\mathrm{P}=0$. & & \\
\hline & & 220 & & & & 367 & & 899 & & \\
\hline & & 236 & & & & 449 & & 1038 & & \\
\hline & & 310 & & & & 432 & & 1014 & & \\
\hline & & 317 & & & & 501 & & 1155 & & \\
\hline
\end{tabular}

Table.3 Effect of tillage, crop residue and supplemental drip irrigation on dry matter production $\left(\mathrm{kg} \mathrm{ha}^{-1}\right)$ of maize 2013

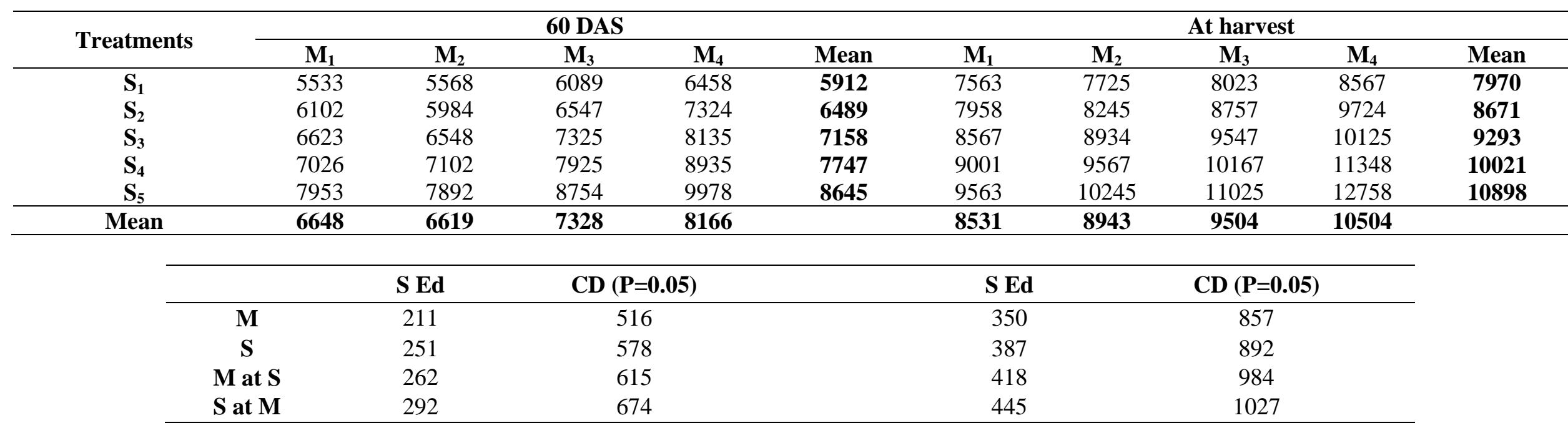


Table.4 Interaction effect of tillage practice with crop residue and supplemental irrigation through drip system on microbial population ( $\mathrm{cfu} \mathrm{g}^{-1}$ of soil) of maize at 60 DAS during 2012

\begin{tabular}{|c|c|c|c|c|c|c|c|c|c|c|c|c|c|c|c|}
\hline \multirow{2}{*}{ Treatments } & \multicolumn{4}{|c|}{ Bacteria $\times 10^{5} \mathrm{cfu} \mathrm{g}^{-1}$ of soil } & \multicolumn{6}{|c|}{ Fungi $x 10^{3} \mathrm{cfu} \mathrm{g}^{-1}$ of soil } & \multicolumn{5}{|c|}{ Actinomycetes x $10^{4} \mathrm{cfu} \mathrm{g}^{-1}$ of soil } \\
\hline & $\mathrm{T}_{1}$ & $\mathrm{~T}_{2}$ & $\mathrm{~T}_{3}$ & $\mathrm{~T}_{4}$ & Mean & $\mathrm{T}_{1}$ & $\mathrm{~T}_{2}$ & $\mathrm{~T}_{3}$ & $\mathrm{~T}_{4}$ & Mean & $\mathrm{T}_{1}$ & $\mathrm{~T}_{2}$ & $\mathrm{~T}_{3}$ & $\mathrm{~T}_{4}$ & Mean \\
\hline $\mathrm{S}_{1}$ & 62.5 & 59.0 & 65.6 & 67.1 & 63.6 & 22.0 & 21.0 & 24.0 & 24.5 & 22.9 & 12.0 & 14.3 & 16.4 & 18.5 & 15.3 \\
\hline $\mathrm{S}_{2}$ & 64.6 & 62.2 & 67.5 & 69.8 & 66.0 & 23.6 & 22.5 & 25.6 & 26.2 & 24.5 & 13.5 & 15.6 & 17.3 & 21.2 & 16.9 \\
\hline $\mathrm{S}_{3}$ & 65.6 & 64.5 & 70.2 & 71.3 & 67.9 & 23.0 & 23.9 & 27.5 & 29.3 & 25.9 & 13.6 & 16.1 & 18.2 & 23.5 & 17.9 \\
\hline $\mathrm{S}_{4}$ & 65.7 & 66.5 & 71.2 & 74.5 & 69.5 & 24.6 & 24.8 & 28.1 & 30.2 & 26.9 & 14.1 & 16.5 & 19.0 & 24.6 & 18.6 \\
\hline $\mathrm{S}_{5}$ & 66.2 & 65.1 & 72.4 & 75.5 & 69.8 & 25.1 & 24.6 & 27.8 & 30.8 & 27.1 & 14.0 & 17.1 & 21.2 & 26.0 & 19.6 \\
\hline \multirow[t]{2}{*}{ Mean } & 64.9 & 63.5 & 69.4 & 71.6 & & 23.7 & 23.4 & 26.6 & 28.2 & & 13.4 & 15.9 & 18.4 & 22.8 & \\
\hline & SEd & \multicolumn{3}{|c|}{$\mathrm{CD}(\mathrm{P}=0.05)$} & & SEd & \multicolumn{3}{|c|}{$\mathrm{CD}(\mathrm{P}=0.05)$} & & \multicolumn{2}{|c|}{ SEd } & \multicolumn{3}{|c|}{$\mathrm{CD}(\mathrm{P}=0.05)$} \\
\hline $\mathrm{T}$ & 1.57 & \multicolumn{2}{|c|}{3.83} & & & 0.62 & \multicolumn{3}{|c|}{1.51} & & \multicolumn{2}{|c|}{0.48} & \multicolumn{3}{|c|}{1.18} \\
\hline $\mathrm{S}$ & 1.73 & \multicolumn{2}{|c|}{3.98} & & & 0.66 & \multicolumn{3}{|c|}{1.51} & & \multicolumn{2}{|c|}{0.50} & \multicolumn{3}{|c|}{1.15} \\
\hline $\mathrm{T}$ at $\mathrm{S}$ & 1.79 & \multicolumn{2}{|c|}{4.21} & & & 0.80 & \multicolumn{3}{|c|}{1.89} & & \multicolumn{2}{|c|}{0.60} & \multicolumn{3}{|c|}{1.41} \\
\hline $\mathrm{S}$ at $\mathrm{T}$ & 1.92 & \multicolumn{2}{|c|}{4.42} & & & 0.82 & \multicolumn{3}{|c|}{1.90} & & \multicolumn{2}{|c|}{0.60} & \multicolumn{3}{|c|}{1.38} \\
\hline
\end{tabular}

Table.5 Interaction effect of tillage practice with crop residue and supplemental irrigation through drip system on microbial population ( $\mathrm{cfu} \mathrm{g}^{-1}$ of soil) of maize at harvest during 2012

\begin{tabular}{|c|c|c|c|c|c|c|c|c|c|c|c|c|c|c|c|}
\hline \multirow[t]{2}{*}{ Treatments } & \multicolumn{5}{|c|}{$\begin{array}{c}\text { Bacteria } \\
\times 10^{5} \mathrm{cfu} \mathrm{g}^{-1} \text { of soil }\end{array}$} & \multicolumn{5}{|c|}{$\begin{array}{c}\text { Fungi } \\
\times 10^{3} \mathrm{cfu} \mathrm{g}^{-1} \text { of soil }\end{array}$} & \multicolumn{5}{|c|}{$\begin{array}{c}\text { Actinomycetes } \\
\times 10^{4} \mathrm{cfu} \mathrm{g}^{-1} \text { of soil }\end{array}$} \\
\hline & $\mathrm{T}_{1}$ & $\mathrm{~T}_{2}$ & $\mathrm{~T}_{3}$ & $\mathrm{~T}_{4}$ & Mean & $\mathrm{T}_{1}$ & $\mathrm{~T}_{2}$ & $\mathrm{~T}_{3}$ & $\mathrm{~T}_{4}$ & Mean & $\mathrm{T}_{1}$ & $\mathrm{~T}_{2}$ & $\mathrm{~T}_{3}$ & $\mathrm{~T}_{4}$ & Mean \\
\hline $\mathrm{S}_{1}$ & 11.9 & 12.9 & 15.4 & 16.4 & 14.1 & 9.3 & 10.7 & 11.8 & 12.3 & 11.0 & 7.5 & 9.2 & 11.2 & 13.3 & 10.3 \\
\hline $\mathrm{S}_{2}$ & 12.7 & 14.0 & 16.0 & 19.4 & 15.5 & 10.3 & 10.8 & 12.1 & 12.1 & 11.3 & 8.3 & 9.8 & 11.6 & 13.6 & 10.8 \\
\hline $\mathrm{S}_{3}$ & 13.0 & 13.9 & 17.8 & 20.0 & 16.2 & 10.7 & 11.3 & 12.2 & 13.1 & 11.8 & 8.5 & 10.3 & 12.4 & 13.9 & 11.3 \\
\hline $\mathrm{S}_{4}$ & 12.7 & 15.1 & 19.0 & 21.0 & 17.0 & 11.0 & 11.5 & 13.2 & 13.6 & 12.3 & 8.9 & 11.2 & 14.3 & 15.4 & 12.5 \\
\hline $\mathrm{S}_{5}$ & 11.9 & 15.0 & 20.0 & 21.5 & 17.1 & 11.2 & 12.1 & 14.1 & 15.2 & 13.2 & 9.5 & 12.1 & 16.5 & 17.6 & 13.9 \\
\hline \multirow[t]{2}{*}{ Mean } & 12.4 & 14.2 & 17.6 & 19.7 & & 10.5 & 11.3 & 12.7 & 13.3 & & 8.5 & 10.5 & 13.2 & 14.8 & \\
\hline & & SEd & \multicolumn{3}{|c|}{$\mathrm{CD}(\mathrm{P}=0.05)$} & SEd & \multicolumn{3}{|c|}{$\mathrm{CD}(\mathrm{P}=0.05)$} & & \multicolumn{2}{|c|}{ SEd } & \multicolumn{3}{|c|}{$\mathrm{CD}(\mathrm{P}=0.05)$} \\
\hline \multicolumn{2}{|l|}{$\mathrm{T}$} & 0.46 & \multicolumn{3}{|c|}{1.12} & 0.32 & \multicolumn{3}{|c|}{0.79} & & \multicolumn{2}{|c|}{0.26} & \multicolumn{3}{|c|}{0.64} \\
\hline \multicolumn{2}{|l|}{$\mathrm{S}$} & 0.54 & \multicolumn{3}{|c|}{1.25} & 0.37 & \multicolumn{3}{|c|}{0.85} & & \multicolumn{2}{|c|}{0.37} & \multicolumn{3}{|c|}{0.84} \\
\hline \multicolumn{2}{|l|}{$\mathrm{T}$ at $\mathrm{S}$} & 0.56 & \multicolumn{3}{|c|}{1.31} & 0.38 & \multicolumn{3}{|c|}{0.89} & & \multicolumn{2}{|c|}{0.38} & \multicolumn{3}{|c|}{0.89} \\
\hline \multicolumn{2}{|l|}{$\mathrm{S}$ at $\mathrm{T}$} & 0.62 & \multicolumn{2}{|c|}{1.43} & & 0.42 & \multicolumn{3}{|c|}{0.97} & & \multicolumn{2}{|c|}{0.46} & \multicolumn{3}{|c|}{1.05} \\
\hline
\end{tabular}


Table.6 Interaction effect of tillage practice with crop residue and supplemental irrigation through drip system on soil microbial population ( $\mathrm{cfu} \mathrm{g}^{-1}$ of soil) of maize at 60 DAS during 2013

\begin{tabular}{|c|c|c|c|c|c|c|c|c|c|c|c|c|c|c|c|}
\hline \multirow{2}{*}{ Treatments } & \multicolumn{5}{|c|}{ Bacteria $\times 10^{5} \mathrm{cfu} \mathrm{g}^{-1}$ of soil } & \multicolumn{5}{|c|}{ Fungi x $10^{3} \mathrm{cfu} \mathrm{g}^{-1}$ of soil } & \multicolumn{5}{|c|}{ Actinomycetes $\times 10^{4} \mathrm{cfu} \mathrm{g}^{-1}$ of soil } \\
\hline & $\mathrm{T}_{1}$ & $\mathrm{~T}_{2}$ & $\mathrm{~T}_{3}$ & $\mathrm{~T}_{4}$ & Mean & $\mathrm{T}_{1}$ & $\mathrm{~T}_{2}$ & $\mathrm{~T}_{3}$ & $\mathrm{~T}_{4}$ & Mean & $\mathrm{T}_{1}$ & $\mathrm{~T}_{2}$ & $\mathrm{~T}_{3}$ & $\mathrm{~T}_{4}$ & Mean \\
\hline $\mathrm{S}_{1}$ & 66.1 & 69.2 & 75.3 & 77.0 & 71.9 & 20.0 & 23.6 & 25.6 & 28.4 & 24.4 & 10.3 & 13.8 & 15.4 & 17.0 & 14.1 \\
\hline $\mathrm{S}_{2}$ & 69.3 & 72.6 & 79.2 & 82.2 & 75.8 & 23.4 & 26.5 & 28.6 & 33.2 & 27.9 & 12.1 & 14.5 & 16.3 & 20.3 & 15.8 \\
\hline $\mathrm{S}_{3}$ & 70.2 & 73.2 & 81.3 & 84.6 & 77.3 & 24.2 & 27.5 & 29.6 & 36.5 & 29.5 & 12.2 & 15.5 & 17.2 & 21.2 & 16.5 \\
\hline $\mathrm{S}_{4}$ & 70.0 & 75.5 & 81.0 & 86.2 & 78.2 & 23.2 & 27.2 & 30.1 & 37.5 & 29.5 & 13.1 & 15.2 & 17.3 & 23.5 & 17.3 \\
\hline $\mathrm{S}_{5}$ & 74.4 & 79.2 & 83.5 & 87.3 & 81.1 & 24.0 & 27.0 & 30.5 & 37.6 & 29.8 & 13.4 & 15.9 & 17.2 & 24.5 & 17.7 \\
\hline \multirow[t]{2}{*}{ Mean } & $\mathbf{7 0 . 0}$ & 73.9 & 80.1 & 83.5 & & 23.0 & 26.4 & 28.9 & 34.6 & & 12.2 & 15.0 & 16.7 & 21.3 & \\
\hline & $\mathrm{SEd}$ & \multicolumn{3}{|c|}{$\mathrm{CD}(\mathrm{P}=0.05)$} & & \multicolumn{2}{|c|}{ SEd } & \multicolumn{3}{|c|}{$\mathrm{CD}(\mathrm{P}=0.05)$} & \multicolumn{3}{|c|}{ SEd } & \multicolumn{2}{|c|}{$\mathrm{CD}(\mathrm{P}=0.05)$} \\
\hline $\mathrm{T}$ & 1.61 & \multicolumn{3}{|c|}{3.93} & & \multicolumn{2}{|c|}{0.70} & \multicolumn{2}{|c|}{1.72} & & \multicolumn{3}{|c|}{0.36} & \multicolumn{2}{|c|}{0.88} \\
\hline $\mathrm{S}$ & 1.87 & \multicolumn{3}{|c|}{4.30} & & \multicolumn{2}{|c|}{0.79} & \multicolumn{2}{|c|}{1.81} & & \multicolumn{3}{|c|}{0.46} & \multicolumn{2}{|c|}{1.06} \\
\hline $\mathrm{T}$ at $\mathrm{S}$ & 1.77 & \multicolumn{3}{|c|}{4.17} & & \multicolumn{2}{|c|}{0.78} & \multicolumn{2}{|c|}{1.84} & & \multicolumn{3}{|c|}{0.48} & \multicolumn{2}{|c|}{1.13} \\
\hline $\mathrm{S}$ at $\mathrm{T}$ & 2.00 & \multicolumn{3}{|c|}{4.62} & & \multicolumn{2}{|c|}{0.86} & \multicolumn{2}{|c|}{1.97} & & \multicolumn{3}{|c|}{0.56} & \multicolumn{2}{|c|}{1.28} \\
\hline
\end{tabular}

Table.7 Interaction effect of tillage practice with crop residue and supplemental irrigation through drip system on microbial population ( $\mathrm{cfu} \mathrm{g}^{-1}$ of soil) of maize at harvest during 2013

\begin{tabular}{|c|c|c|c|c|c|c|c|c|c|c|c|c|c|c|c|}
\hline \multirow{2}{*}{ Treatments } & \multicolumn{5}{|c|}{ Bacteria $\times 10^{5} \mathrm{cfu} \mathrm{g}^{-1}$ of soil } & \multicolumn{5}{|c|}{ Fungi $x 10^{3} \mathrm{cfu} \mathrm{g}^{-1}$ of soil } & \multicolumn{5}{|c|}{ Actinomycetes $\times 10^{4} \mathrm{cfu} \mathrm{g}^{-1}$ of soil } \\
\hline & $\mathrm{T}_{1}$ & $\mathrm{~T}_{2}$ & $\mathrm{~T}_{3}$ & $\mathrm{~T}_{4}$ & Mean & $\mathrm{T}_{1}$ & $\mathrm{~T}_{2}$ & $\mathrm{~T}_{3}$ & $\mathrm{~T}_{4}$ & Mean & $\mathrm{T}_{1}$ & $\mathrm{~T}_{2}$ & $\mathrm{~T}_{3}$ & $\mathrm{~T}_{4}$ & Mean \\
\hline $\mathrm{S}_{1}$ & 18.0 & 20.1 & 24.5 & 26.3 & 22.2 & 8.3 & 10.2 & 12.2 & 13.4 & 11.0 & 7.0 & 10.2 & 11.9 & 13.9 & 10.8 \\
\hline $\mathrm{S}_{2}$ & 18.6 & 21.3 & 25.5 & 27.3 & 23.2 & 8.3 & 10.0 & 12.5 & 14.2 & 11.3 & 8.1 & 11.2 & 12.5 & 13.5 & 11.3 \\
\hline $\mathrm{S}_{3}$ & 19.5 & 21.2 & 25.6 & 27.2 & 23.4 & 9.6 & 11.6 & 12.6 & 15.6 & 12.4 & 8.3 & 13.2 & 12.6 & 14.1 & 12.1 \\
\hline $\mathrm{S}_{4}$ & 20.6 & 24.2 & 26.3 & 27.0 & 24.5 & 10.3 & 11.6 & 13.5 & 15.3 & 12.7 & 8.5 & 13.1 & 13.2 & 14.6 & 12.4 \\
\hline $\mathrm{S}_{5}$ & 22.2 & 25.5 & 27.1 & 28.7 & 25.9 & 11.2 & 12.1 & 14.5 & 15.9 & 13.4 & 9.2 & 13.2 & 14.1 & 15.2 & 12.9 \\
\hline \multirow[t]{2}{*}{ Mean } & 19.8 & 22.5 & 25.8 & 27.3 & & 9.5 & 11.1 & 13.1 & 14.9 & & 8.2 & 12.2 & 12.9 & 14.3 & \\
\hline & SEd & \multicolumn{4}{|c|}{$\mathrm{CD}(\mathrm{P}=0.05)$} & SEd & \multicolumn{3}{|c|}{$\mathrm{CD}(\mathrm{P}=0.05)$} & & \multicolumn{3}{|c|}{ SEd } & \multicolumn{2}{|c|}{$\mathrm{CD}(\mathrm{P}=0.05$} \\
\hline $\mathrm{T}$ & 0.61 & \multicolumn{3}{|c|}{1.50} & & 0.32 & \multicolumn{3}{|c|}{0.78} & & \multicolumn{3}{|c|}{0.28} & \multicolumn{2}{|r|}{0.68} \\
\hline $\mathrm{S}$ & 0.63 & \multicolumn{3}{|c|}{1.45} & & 0.33 & \multicolumn{3}{|c|}{0.77} & & \multicolumn{3}{|c|}{0.28} & \multicolumn{2}{|r|}{0.64} \\
\hline $\mathrm{T}$ at $\mathrm{S}$ & 0.84 & \multicolumn{3}{|c|}{1.98} & & 0.46 & \multicolumn{3}{|c|}{1.08} & & \multicolumn{3}{|c|}{0.37} & \multicolumn{2}{|r|}{0.88} \\
\hline $\mathrm{S}$ at $\mathrm{T}$ & 0.84 & \multicolumn{3}{|c|}{1.94} & & 0.46 & \multicolumn{3}{|c|}{1.06} & & \multicolumn{3}{|c|}{0.37} & \multicolumn{2}{|r|}{0.85} \\
\hline
\end{tabular}


Table.8 Effect of tillage, crop residue and supplemental drip irrigation on yield attributes and yield of maize 2012

\begin{tabular}{|c|c|c|c|c|c|c|c|c|c|c|c|c|c|c|c|}
\hline \multirow{2}{*}{ Treatments } & \multicolumn{5}{|c|}{ Cob length $(\mathrm{cm})$} & \multicolumn{5}{|c|}{ Cob weight (g) } & \multicolumn{5}{|c|}{ Yield $\left(\mathrm{kg} \mathrm{ha}^{-1}\right)$} \\
\hline & $\mathbf{M}_{1}$ & $\mathbf{M}_{2}$ & $\mathbf{M}_{3}$ & $\mathbf{M}_{4}$ & Mean & $\mathbf{M}_{1}$ & $\mathbf{M}_{2}$ & $\mathbf{M}_{3}$ & $\mathbf{M}_{4}$ & Mean & $\mathbf{M}_{1}$ & $\mathbf{M}_{2}$ & $\mathbf{M}_{3}$ & $\mathbf{M}_{4}$ & Mean \\
\hline $\mathbf{S}_{1}$ & 16.7 & 16.9 & 17.4 & 17.6 & 17.2 & 170 & 174 & 179 & 183 & 177 & 5034 & 5800 & 6783 & 6810 & 6107 \\
\hline $\mathbf{S}_{2}$ & 16.6 & 17.0 & 17.9 & 18.1 & 17.4 & 174 & 179 & 184 & 190 & 182 & 5100 & 5890 & 7077 & 7328 & 6349 \\
\hline $\mathbf{S}_{\mathbf{3}}$ & 17.3 & 17.6 & 18.0 & 19.5 & 18.1 & 177 & 183 & 189 & 193 & 186 & 6103 & 6857 & 7350 & 7465 & 6944 \\
\hline $\mathbf{S}_{4}$ & 17.0 & 18.2 & 19.0 & 19.3 & 18.4 & 181 & 189 & 200 & 204 & 194 & 6670 & 6945 & 7421 & 7724 & 7190 \\
\hline $\mathbf{S}_{\mathbf{5}}$ & 18.2 & 19.2 & 20.2 & 23.2 & 20.2 & 188 & 197 & 209 & 221 & 204 & 6745 & 7321 & 7945 & 8345 & 7589 \\
\hline \multirow[t]{2}{*}{ Mean } & 17.2 & 17.8 & 18.5 & 19.5 & & 179 & 185 & 192 & 199 & & 5930 & 6563 & 7316 & 7534 & \\
\hline & \multicolumn{3}{|c|}{ S Ed } & \multicolumn{2}{|c|}{$\mathrm{CD}(\mathrm{P}=0.05)$} & S Ed & \multicolumn{3}{|c|}{$\mathrm{CD}(\mathrm{P}=\mathbf{0 . 0 5})$} & \multicolumn{2}{|c|}{ S Ed } & \multicolumn{4}{|c|}{$\mathrm{CD}(\mathrm{P}=\mathbf{0 . 0 5})$} \\
\hline $\mathbf{M}$ & \multicolumn{3}{|c|}{0.54} & \multicolumn{2}{|c|}{1.33} & 4.34 & \multicolumn{3}{|c|}{10.63} & \multicolumn{2}{|c|}{267} & \multicolumn{4}{|c|}{655} \\
\hline $\mathbf{S}$ & \multicolumn{3}{|c|}{0.64} & \multicolumn{2}{|c|}{1.47} & 5.35 & \multicolumn{3}{|c|}{12.33} & \multicolumn{2}{|c|}{282} & \multicolumn{4}{|c|}{651} \\
\hline $\mathbf{M}$ at $\mathbf{S}$ & \multicolumn{3}{|c|}{0.72} & \multicolumn{2}{|c|}{1.69} & 5.35 & \multicolumn{3}{|c|}{12.55} & \multicolumn{2}{|c|}{333} & \multicolumn{4}{|c|}{786} \\
\hline $\mathbf{S}$ at $\mathbf{M}$ & \multicolumn{3}{|c|}{0.78} & \multicolumn{2}{|c|}{1.80} & 6.14 & \multicolumn{3}{|c|}{14.16} & \multicolumn{2}{|c|}{341} & \multicolumn{4}{|c|}{788} \\
\hline
\end{tabular}

Table.9 Effect of tillage, crop residue and supplemental drip irrigation on yield attributes and yield of maize 2013

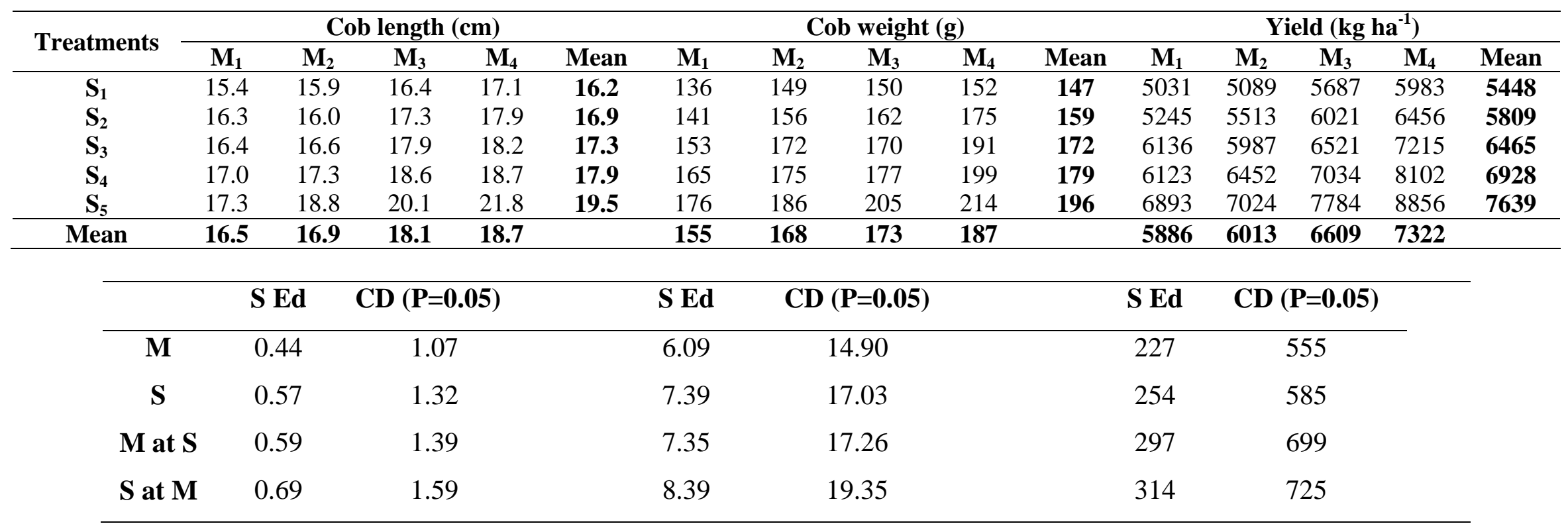


With regard to interaction effect, in a given tillage with crop residue treatment and supplemental drip irrigation, minimum tillage with crop residue $10 \mathrm{t} \mathrm{ha}^{-1}+$ supplemental drip irrigation 10 times registered significantly higher dry matter production at 120 DAS and at harvest during both the years of study.

This might be due to the reason that minimum tillage conserved more soil moisture and crop residues have potential to increase of soil organic matter and nutrient levels, moderation of soil temperature and augmented soil biological activity, which provided better growing environment for increased plant height. Minimum tillage indirectly defines the species composition of the soil microbial community by improving retention of soil moisture and modifying soil temperature (Krupinsky et al., 2002).

\section{Microbiological population}

Tillage and crop residue with supplemental irrigation through drip irrigation obviously influenced the microbe's population.

Soil organic matter distribution, nutrient cycling and microbial activity are influenced by the type and the degree of soil tillage (Salinas-Garcia et al., 2002).

The influence of tillage and supplemental irrigation on the soil biological properties was studied through the assessment of soil microbial population.

Practice of different tillage systems and supplemental irrigation significantly energized the soil microbial load at early stages during both the years of experimentation. The fluctuation in the microbial load in the soil is based on the availability of carbon source in the soil and enhanced microbial activity stimulated by crop residues and manures (Hoflich et al., 2000).

Among tillage and crop residue treatments, minimum tillage with crop residue $10 \mathrm{t} \mathrm{ha}^{-1}$ recorded significantly higher population (Tables 4-7) at 80 and 120 DAS during both the years of study.

Among irrigation practices, supplemental irrigation at 10 times was recorded higher number of bacteria, fungal and actinomycetes at 80 and 120 DAS during both the years than that in without supplemental irrigation plot.

With regard to interaction effect, in a given tillage with crop residue treatment and supplemental drip irrigation, minimum tillage with crop residue $10 \mathrm{t} \mathrm{ha}^{-1}+$ supplemental drip irrigation 10 times had higher influence on the population of bacteria, fungal and actinomycetes at 80 and 120 DAS during both the years than others both the years of study.

\section{Effect of treatments on yield attributes and yield}

Yield attribute like cob length and cob weight and yield of maize was significantly influenced by tillage, crop residue and supplemental irrigation through drip irrigation.

Among tillage and crop residue treatments, minimum tillage with crop residue $10 \mathrm{t} \mathrm{ha}^{-1}$ recorded significantly higher cob length and cob weight and yield (Tables 8 and 9) (19.5 cm cob length, $199 \mathrm{~g}$ cob weight, $7534 \mathrm{~kg} \mathrm{ha}^{-1}$ and $18.7 \mathrm{~cm}$ cob length, $187 \mathrm{~g}$ cob weight, $7322 \mathrm{~kg} \mathrm{ha}{ }^{-1}$ during 2012 and 2013, respectively) Regarding irrigation practices, supplemental irrigation at 10 times was recorded higher cob length and cob weight and yield $(20.2 \mathrm{~cm}$ cob length, $204 \mathrm{~g}$ cob weight, $7589 \mathrm{~kg} \mathrm{ha}^{-1}$ and $19.5 \mathrm{~cm}$ cob length, $196 \mathrm{~g}$ cob weight, $7639 \mathrm{~kg} \mathrm{ha}^{-1}$ during 2012 
and 2013, respectively) than that in without supplemental irrigation plot.

With regard to interaction effect, in a given tillage with crop residue treatment and supplemental drip irrigation, minimum tillage with crop residue $10 \mathrm{t} \mathrm{ha}^{-1}+$ supplemental drip irrigation 10 times registered laudably higher cob length and number of bolls per plant and yield during both the years.

This might be due to the reason that the minimum tillage plots had more main stem nodes, numbers of fruiting sites than those on conventional tillage. Consequently, the number of bolls retained was greater under the minimum tillage than under the conventional tillage system. Enhanced boll retention in the minimum tillage treatments could be due to other factors such as less competition from weeds, differences in nutrient supply and conserved soil moisture. Greater boll numbers on the minimum tillage plots contributed to yield improvements compared to the conventional tillage (Blaise. 2011). Tolessa Debele (2011) concluded that minimum tillage with residue retention increased yield particularly when crop faced terminal drought.

From these experiments, it is concluded that practicing of minimum tillage and application of crop residue at $10 \mathrm{t} \mathrm{ha}^{-1}+$ supplemental drip irrigation 10 times was found to be the promising agronomic practice for enhancing growth, microbial population and productivity of hybrid maize under rainfed situation.

\section{References}

Saviozzi A., Levi-Minzi R., Cardelli R., Riffaldi R. A comparison of soil quality in adjacent cultivated, forest and native grassland soils. Plant Soil 233, 251, 2001.

Kandeler E., Tscherko D., Spiegel H. Long- term monitoring of microbial biomass, $\mathrm{N}$ mineralization and enzyme activities of a Chernozem under different tillage management. Biol. Fertil. Soils 28, 343, 1999.

Helgason B.L., Walley F.L., Germida J. Fungal and bacterial abundance in longterm no-till and intensive till soils of the northern great plains. Soil Sci. Soc. Am. J., 73, (1), 120, 2009.

Klavdivko E. Tillage systems and soil ecology. Soil Till. Res. 61, 61, 2001.

Soon Y.K., Clayton G.W., Rice W.A. Tillage and previous crop effects on dynamics of nitrogen in a wheat-soil system. Agron. J. 93, 842, 2001.

Acosta-Martinez V., Zobeck T., Gill T.E., Kennedy A.C. Enzyme activities and microbial community structure in semiarid agricultural soils. Biol. Fertil. Soils 38, 216, 2003.

Franzluebbers A.J., Hons F.M., Zuberer A.D. Tillage and crop effects on seasonal soil carbon and nitrogen dynamics. Soil Sci. Soc. Am. J. 59, (6), 1618, 1995.

Frey, S.D.; Elliott, E.T.; Paustian, K. Bacterial and fungal abundance and biomass in conventional and no-tillage agroecosystems along two climatic gradients. Soil Biol. Biochem. 1999, 31, 573-585.

Oweis, T. and Hachum, A. 2006. Water management in rainfed agriculture investing in supplemental irrigation. In: Agricultural Water Sourcebook: Shaping the Future of Water for Agriculture. The World Bank, Washington, DC, USA, pp. 206-213.

Krupinsky, J.M., Bailey, K.L., McMullen, M.P., Gossen, B.D., Turkingtond, T.K., 2002. Managing plant disease risk in diversified cropping systems. Agron. J. 94, 198-209.

Salinas-Garcıa, J.R., Velazquez-Garcıa, J.J., Gallardo-Valdez, M., Diaz-Mederos, P., Caballero-Hernandez, F., Tapia-Vargas, 
L.M., Rosales-Robles, E., 2002. Tillage effects on microbial biomass and nutrient distribution in soils under rainfed corn production in central-western Mexico. Soil Till. Res. 66, 143-152.

Hoflich, G., M. Tauschke, G. Kuhn and J. Rogasik. 2000. Influence of agricultural crops and fertilization on microbial activity and micro-organisms in the rhizosphere. J. Agron. Crop Sci., 184: 49-54.

Blaise, D. 2011. Tillage and green manure effects on Bt transgenic cotton (Gossypium hirsutum L.) hybrid grown on rainfed Vertisols of central India. Soil Tillage Res. 114: 86-96.

Tolessa Debele. 2011. The effect of minimum and conventional tillage systems on maize grain yield and soil fertility in western Ethiopia. $5^{\text {th }}$ World Congress on Conservation Agriculture Incorporating $3^{\text {rd }}$ Farming System Conference, 26-29 September 2011, Brisbane, Australia.

\section{How to cite this article:}

Saravanan, M. 2017. Effect of Tillage Practices and Supplemental Irrigation Options on Growth, Yield and Soil Biological Properties of Hybrid Maize. Int.J.Curr.Microbiol.App.Sci. 6(8): 2315-2325. doi: https://doi.org/10.20546/ijcmas.2017.608.273 